\title{
Automatic Segmentation of Left Atrial Geometry from Contrast-Enhanced Magnetic Resonance Images using a Probabilistic Atlas
}

\author{
R. Karim ${ }^{1,3}$, C. Juli ${ }^{2}$, L. Malcolme-Lawes ${ }^{3}$, D. Wyn-Davies ${ }^{3}$, P. \\ Kanagaratnam $^{3}$, N. Peters ${ }^{3}$, and D. Rueckert ${ }^{1}$ \\ 1 Department of Computing, Imperial College London, 180 Queen's Gate, South \\ Kensington, London SW7 2AZ \\ 2 Imaging Department, St. Mary's Hospital, Praed Street, London W2 1NY. \\ 3 National Heart and Lung Institute, Imperial College London, 180 Queen's Gate, \\ South Kensington, London SW7 2AZ \\ rkarim@imperial.ac.uk
}

\begin{abstract}
Left atrium segmentation and the extraction of its geometry remains a challenging problem despite of existing approaches. It is a clinically-relevant important problem with an increasing interest as more research into the mechanism of atrial fibrillation and its recurrence process is undertaken. Contrast-Enhanced (CE) Magnetic Resonance Angiography (MRA) produces excellent images for extracting the atrial geometry. Nevertheless, the variable anatomy of the atrium poses significant challenge for segmentation. To overcome the inherent difficulties with this segmentation, we propose a technique that utilizes the Voronoi subdivision framework for the segmentation. In addition, the segmentation is based on the minimization of a Markov Random Field based energy functional defined within the Voronoi framework. The method also incorporates anatomical priors in the form of a probabilistic atlas. We show how the model is efficient in segmenting atrium images by comparing results from manual segmentations.
\end{abstract}

Key words: Segmentation, Left atrium, Graph Cuts, Magnetic Resonance Angiography

\section{Introduction}

Atrial fibrillation (AF) is a clinically challenging cardiac arrhythmia that occurs in epidemic proportions with an increasing prevalence worldwide even after accounting for the aging population [10]. The electrical isolation of AF-causing circuits with catheter ablation has emerged as an interventional treatment for AF. High radiofrequency energy inducing thermal damage creates lesions or scars in the left atrium, whereby the electrical conduction pathways of abnormal circuits are disrupted. AF recurrence is not uncommon in patients who underwent ablation treatment and is generally caused by the rejuvenation of scarred tissues. Understanding the temporal process of scar formation and whether scar 
reduces or expands over time has become important in determining whether some patients are more susceptible to AF recurrence than others [11].

The quantification of the extent of left atrial scar tissue has now become possible due to recent advancements in imaging techniques. However, for assessing scar information it is important to visualize them on the atrial geometry. Contrast-enhanced Magnetic Resonance Angiography provides good quality imaging data for segmenting the atrial anatomy. Previous work on the segmentation of the left atrium include segmentation from MRA/CTA [1,4], CT [7] and X-ray angiography [8]. Our proposed method adopts the Voronoi framework of $[1,4]$. However, the segmentation method is based on the minimization of an energy functional with a spatial and intensity prior. With a high degree of variability in the anatomy of the atrium, a probabilistic atlas created from training images makes the segmentation approach more robust.

\section{Building a 3D Probabilistic Atlas of the Atrium}

\subsection{Segmentation of Training Images}

The variability of atrial anatomy is documented in the clinical literature [5]. The number and branching patterns of pulmonary vein drainages to the left atrium are known to vary across patient subjects. For modelling this variable anatomy, 20 left atrium images were manually segmented from contrast-enhanced Magnetic Resonance Angiography (MRA) images. Noting the possible anatomical variations from [5], we classified our training left atrium images into the different anatomical groups. The most common anatomical variations found were: 1 ) two drainages to the right and left sides (4-drainage), and 2) a single drainage to the left and two drainages to the right sides (3-drainage). After segmenting the images, shape based interpolation [12] was used to resample the images to isotropic voxels of size $1 \mathrm{~mm} \times 1 \mathrm{~mm} \times 1 \mathrm{~mm}$.

\subsection{Registration of Training Images}

The training images from each anatomical group were registered to a common co-ordinate frame. A left atrium image from each anatomical group was selected as the target image for that group. It was ensured that the atrium selected as reference was a good representative of the anatomy, based on an expert radiologist's opinion. Each training image was then registered to its respective group's reference image. For registration, landmark points were selected on each training atrium: 3 or 4 points, depending on the anatomy, selected at the centre of the opening of the pulmonary drainage and a single point at the centre of the atrial chamber. An affine registration allowing 9 degrees of freedom were used to align the atria and initialize the final registration process. Using the marching cubes algorithm [2], a dense triangulation (pseudo-landmarks) of the boundary surfaces of each segmented atria were generated. The correspondence between each pseudo-landmark was then achieved using a surface based registration method using B-Splines [9]. This aligned all atria images to its respective reference anatomical shape. 


\subsection{Atlas Construction}

A probabilistic atlas for each anatomical group is constructed from the aligned atria images. The atlas is formed by averaging segmentations for each group. A blurring of the segmentations with a Gaussian kernel prior to averaging is needed. With a relatively small population of training images, Gaussian blurring compensates by alleviating undesirable sharp transitions in the probability map. We selected a Gaussian kernel with width 10.0. Too small a kernel creates sharp transitions in foreground probabilities and too large a kernel can suppress the drainage networks in the atlas. Fig. 1 shows the probabilistic atlas for the 4drainage network.
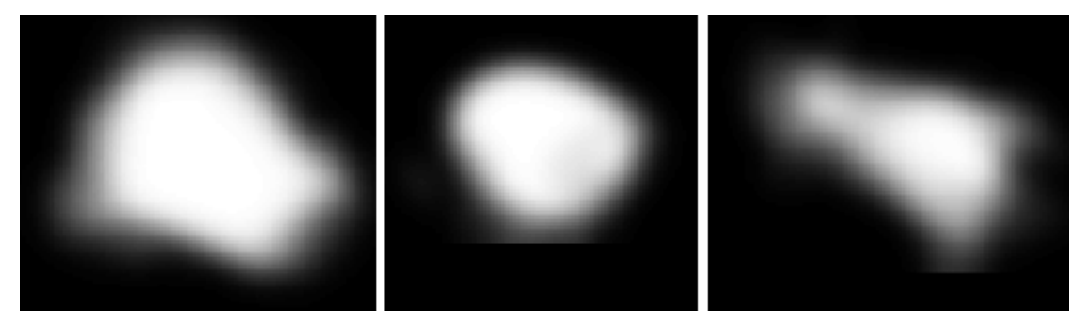

Fig. 1. The probabilistic atlas for the 4-drainage showing transverse (left), sagittal (middle) and coronal (right) planes.

\section{The Segmentation Framework}

\subsection{Voronoi Tessellation of the Distance Transform Space}

The left atrium in CE-MRA images is connected to neighbouring structures via narrow junctions. These junctions were exploited in $[1,4]$ by making the appropriate cuts and separating the atrium from the rest of the image. For finding narrow junctions within a binary image, a Voronoi Tessellation of the Euclidean Distance Transform (EDT) image is computed.

Each Voronoi cell is analogous to a maximally inscribed sphere, where a narrow junction between two structures is analogous to a smaller sphere between two larger spheres. Although the technique performs well on contrast-enhanced images of the atrium, with no anatomical priors incorporated into the model, this rather ad-hoc approach is unable to segment images that are not contrastenhanced. In addition to this, it requires manual interaction as it relies heavily on a merging-threshold parameter. We adopt the same Voronoi framework for segmentation. However, we incorporate an anatomical prior with the probabilistic atlas. In addition to this, we use a MRF based energy function which is optimized using graph-cuts. 
The left atrium blood pool in the CE-MRA consists of structures other than the atrium, such as the aorta and pulmonary arteries. This blood pool is extracted using a region-growing technique with automatic Otsu thresholding [13]. The EDT of this blood pool is computed. Thus, each pixel in the distance transform is assigned the shortest distance to the left atrium blood pool boundary. Local maxima in this EDT image are assigned centres of the Voronoi cells and thus form the basis of our Voronoi tessellation. By definition, each Voronoi cell only comprise of voxels that are closest to its centre. As in [1], each Voronoi cell has a size and the separating surface between cells are also given a size. The separating surface are shared borders with a neighbouring cell and illustrated in Fig. 3. The sizes of the cells and separating surfaces are simply the computed EDT values of their inner-most point (i.e. centre). Every voxel in the atrium blood pool is assigned a Voronoi cell of which it is a member; this is its closest Voronoi cell. Thus the closest Voronoi cells for each voxel (i.e. cell memberships) can be computed using gradient ascent on the distance transform.
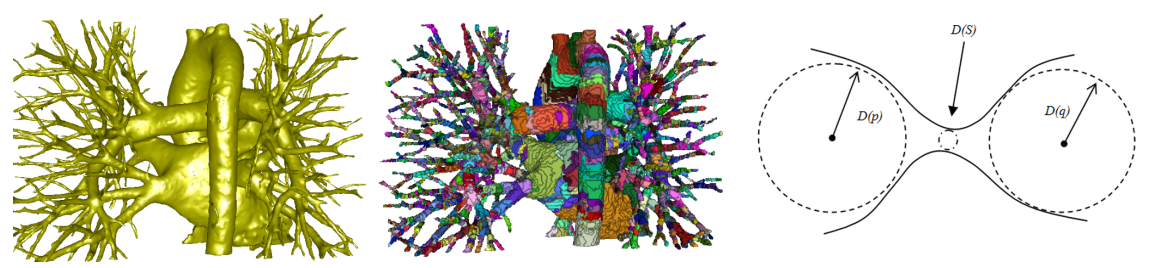

Fig. 2. The blood pool image (left) subdivided into its Voronoi cells (middle), with each cell assigned a random colouring. Right image: Adjacent Voronoi cells are analogous to maximally inscribed circles with a diameter $D(p)$ and $D(q)$. The separating surface also has a diameter $D(\mathcal{S})$

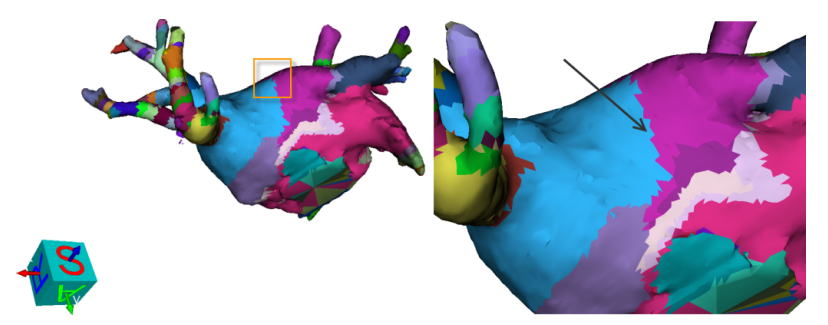

Fig. 3. A left atrium with each Voronoi cell uniquely coloured (left). A close-up of the surface showing shared borders of adjacent or neighbouring cells (right). As cells are $3 \mathrm{D}$ entities, these shared borders are surfaces in 3D. 


\section{$3.2 \quad$ Energy-based Formulation}

With the image subdivided into Voronoi cells $p$, segmenting the left atrium is equivalent to assigning a label $f_{p} \in\{0,1\}$ to every cell in the image $P$. The segmentation is based on the observed intensities in the image and the anatomical prior. MRF provides a sound background to model context dependent image segmentation in which segmentation is formulated as an energy minimization problem. Utilizing our Voronoi framework, an MRF-based energy function is given by:

$$
E_{P}(f)=\lambda \sum_{p \in P} D_{p}\left(f_{p}\right)+\sum_{p, q \in \mathcal{N}} V_{p, q}\left(f_{p}, f_{q}\right)
$$

where the data term $D_{p}\left(f_{p}\right)$ is a function of the observed image data and explains how well label $f_{p}$ can be assigned to the Voronoi cell $p$ based on a probabilistic model. $V_{p, q}\left(f_{p}, f_{q}\right)$ is a smoothness term penalizing discontinuities in a neighbourhood system of Voronoi cells $\mathcal{N}$. As The parameter $\lambda$ weighs the influence of the data and smoothness terms.

\subsection{The Data and Smoothness terms}

The data term in the MRF model is a combination of the spatial and intensity priors. The probabilistic atlas constructed from pre-segmented training images provides each voxel a prior probability of its label being foreground or background. However, as the MRF model is built over the Voronoi framework, the mean probability over all voxels within a Voronoi cell is used as an estimate of its prior probability. The intensity model is derived from the unseen target image. To model the foreground, a Gaussian distribution is used where the mean and variance are determined from the image intensities of voxels (of the unseen target) labelled as foreground by at least $90 \%$ of the training images. In CE-MRA images of good quality, the perfusion of contrast into pulmonary arteries is not as evident as it is in the atrial blood pool. Thus the signal intensity is expected to be higher in the blood pool than in the arteries, thereby making a Gaussian intensity foreground model sensible. The data term for each Voronoi cell is thus given by:

$$
D_{p}\left(f_{p}\right)=\mu \frac{1}{N} \sum_{n_{p} \in p} p\left(f_{p}\right)+P\left(I \mid f_{p}\right)
$$

where $p\left(f_{p}\right)$ is the spatial prior from the atlas averaged over all voxels $n_{p}$ of the Voronoi cell and $P\left(I \mid f_{p}\right)$ is the intensity model. Parameter $\mu$ weighs the influence of the two terms.

The regularizer or the smoothness term in the image relates two adjacent Voronoi cells and allows appropriate cuts to be made in the image. As cuts are sought within narrowing regions in the image, the relative sizes of adjacent Voronoi cells are incorporated into the smoothness term. The relative sizes $\eta$ of two adjacent Voronoi cells $p$ and $q$ is given by $\eta=\min \{D(p), D(q)\}-D\left(\mathcal{S}_{p, q}\right)$. where $D(\cdot)$ is the diameter function of a Voronoi cell and $\mathcal{S}$ is the separating surface or interface between two adjacent cells. Following [1] and [4], we use 
$D(\cdot)$ as the EDT value of the cell and surface centres. Larger values of $\eta$ indicate a Voronoi neighbourhood with a narrowing. The smoothness term $V_{p, q}$ is thus set to a monotonically decreasing function of the relative diameter such as its reciprocal:

$$
V_{p, q}=1 /(1+\eta)
$$

\subsection{Optimization}

The MRF energy model defined in Eq. 1 over the Voronoi framework is a complex functional of the segmentation $\mathbf{f}$. Local optimization methods like simulated annealing are not guaranteed to find a global minimum in polynomial time. However, for MRF functionals of the form defined (Eq. 1) it is possible to find the global optimum using graph cuts [6]. In this method, the functional is converted to a graph $\mathcal{G}=\langle\mathcal{V}, \mathcal{E}\rangle$ with two special $s, t$ terminal nodes representing the foreground and background labels. The segmentation labelling problem is solved by computing the minimum $s$ - $t$ cut.

As each Voronoi cell must be marked as foreground or background in the segmentation problem, a node $v \in \mathcal{V}$ is assigned for each Voronoi cell $p$ in image $P$. Neighbouring cells and thus their nodes are connected by edges $e \in \mathcal{E}$ enforcing continuity in the segmentation. Every node vertex also has edges to the terminal $s$ and $t$ nodes representing the fact that they may be marked either as foreground or background. Edge weights are given by the data term (Eq. 2) for node-to-node connections and by the smoothness term (Eq. 3) for node-toterminal connections. See Fig. 4 for an illustration. By determining the minimum $s-t$ cut in $\mathcal{G}$, the segmentation problem is solved by assigning a label to each node $[3]$.

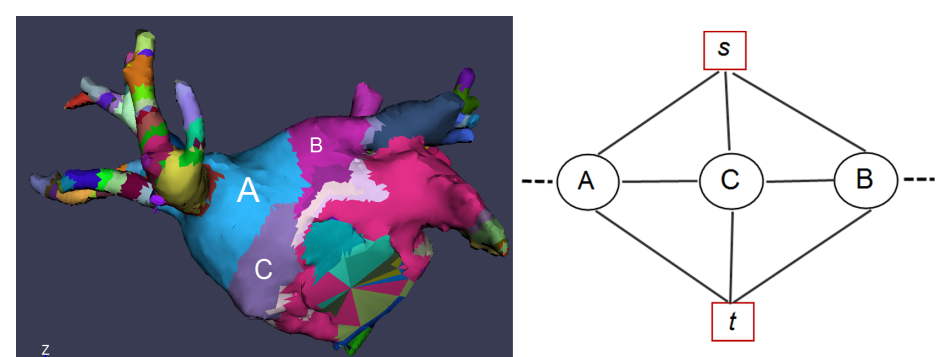

Fig. 4. The Voronoi cells in a segmented left atrium (left). A subset of the graph constructed for optimizing the MRF energy model (right). Note the terminal nodes $s$ and $t$. Only Voronoi cell nodes A, B and C are shown here as an example. Note also that there is a path from A to B, excluded in the diagram for maintaining clarity. 


\section{Experiments}

\subsection{Image Acquisition}

We acquired 30 CE-MRA images of the left atrium from different subjects for our study. All subjects were diagnosed for AF and imaged prior to their ablation. We selected 20 images for our training data, as described in section 2.1. Out of the 20 images, 11 atria images had a 4-drainage network and 9 images with a 3-drainage network. The rest of the images were used for validating our proposed model. All images were acquired at $1.5 \mathrm{~T}$ using a Siemens Avanto scanner. The image size ranges between $250 \times 250 \times 30$ to $380 \times 380 \times 100$ voxels. The slice thickness ranges between 1.0-3.0 $\mathrm{mm}$. Gadovist was used as the contrasting agent.

\subsection{Comparison with manually outlined data}

Segmentations of the atrium were generated using the proposed model on 10 images of the left atrium CE-MRA data. The probabilistic atlas was registered to the unseen instance using affine registration and the minimum graph cut produced the segmentation with no manual intervention. For manual segmentations, for each of the images, 5 slices through the data were selected at equally spaced intervals and the outline of atrium manually delineated in each slice. These slices were selected from the most interesting areas of the image, i.e. in and around the atrial chamber and including the drainages. It is worth noting that the network of veins emanating from the atrium has little clinical importance in the context of ablation procedures, and thus has been excluded from our manual segmentations. Each slice is compared against segmentations from the proposed model using an overlap measure $O_{s}$ defined over the manual $\mathcal{M}$ and computer-assisted segmentation $\mathcal{C}$ as:

$$
O_{s}=\frac{\operatorname{Area}(\mathcal{M} \cap \mathcal{C})}{\operatorname{Area}(\mathcal{M})}
$$

In addition to this, surface visualizations of the segmented left atrial geometry were generated to allow an assessment of whether segmentations included all the drainages to the atrium only as far as the first bifurcation. In visualizing scars that are only made in and around the atrial chamber and drainage openings, this is more clinically relevant than achieving a voxel-wise accuracy of the segmentations.

\subsection{Results}

The results of evaluating the segmentations obtained from the proposed method against manual segmentations are given in Table 1 for each selected slice of image data. Figure 5 compares the mean overlaps found for each subject. The proportion of overlap is between 0 and 1, where an overlap of 1.0 represents complete agreement with the manual segmentation. Note the occasional low overlap values, for example: patient 1 slice 1 , patient 8 slice 1 , patient 8 slice 3 . This is primarily for slices with the atrium-ventricle junction at the mitral valve, 
which is not particularly visible in CE-MRA images. The algorithm relies on the atlas for finding the mitral valve junction and with no explicit model of the valve, this often is an approximation based on the mean atrial shape. However, it is clear from the segmentations that the probabilistic atlas allows the exclusion of the extensive vessel drainage network in angiography images. This produces vessel-free segmentations allowing a clear visualization of the anatomy (see Fig. 6). All segmentations were computed in under 5 secs on a 2 Ghz PC.

\begin{tabular}{|c|c|c|c|c|c|c|c|c|c|c|}
\hline & \multicolumn{10}{|c|}{ Subjects } \\
\hline & 1 & 2 & 3 & 4 & 5 & 6 & 7 & 8 & 9 & 10 \\
\hline slice 1 & 0.50 & 0.00 & 0.84 & 0.99 & 0.78 & 0.99 & 0.85 & 0.31 & 0.68 & 0.66 \\
\hline slice 2 & 0.98 & 0.99 & 0.99 & 0.99 & 0.99 & 0.97 & 0.94 & 0.97 & 0.90 & 0.97 \\
\hline slice 3 & 0.98 & 0.99 & 0.99 & 0.98 & 0.99 & 0.97 & 0.99 & 0.25 & 0.99 & 0.90 \\
\hline slice 4 & 0.98 & 0.99 & 0.99 & 0.99 & 0.99 & 0.99 & 0.99 & 0.98 & 0.99 & 0.99 \\
\hline slice 5 & 0.98 & 0.99 & 0.92 & 0.99 & 0.99 & 0.89 & 0.99 & 0.99 & 0.97 & 0.97 \\
\hline \hline mean & 0.89 & 0.80 & 0.95 & 0.99 & 0.95 & 0.97 & 0.96 & 0.70 & 0.91 & 0.90 \\
\hline
\end{tabular}

Table 1. Overlap between manual segmentation and the proposed method. A value of 1.0 represents complete overlap.

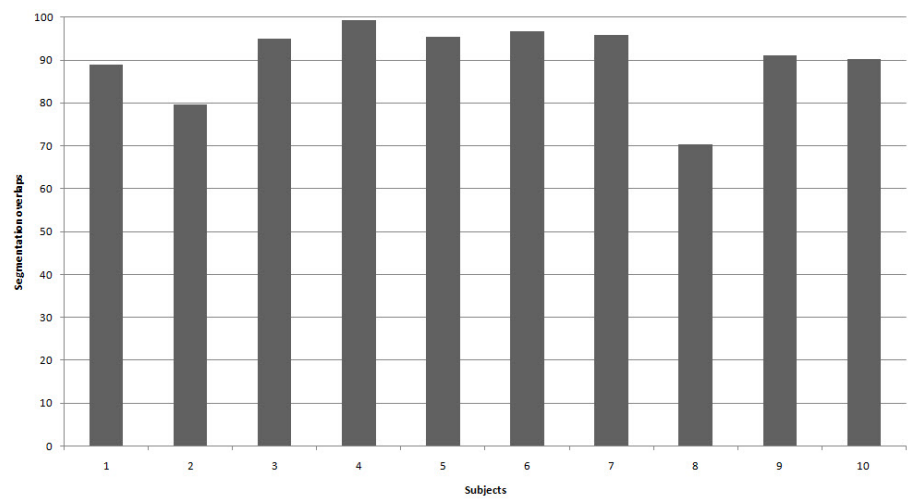

Fig. 5. The mean overlap (between manual and our segmentation) over the selected 5 slices for each of the 10 subjects.

\section{Discussion}

This paper describes a new and novel approach for segmenting the left atrium from CE-MRA images via an energy minimization approach. Existing approaches 

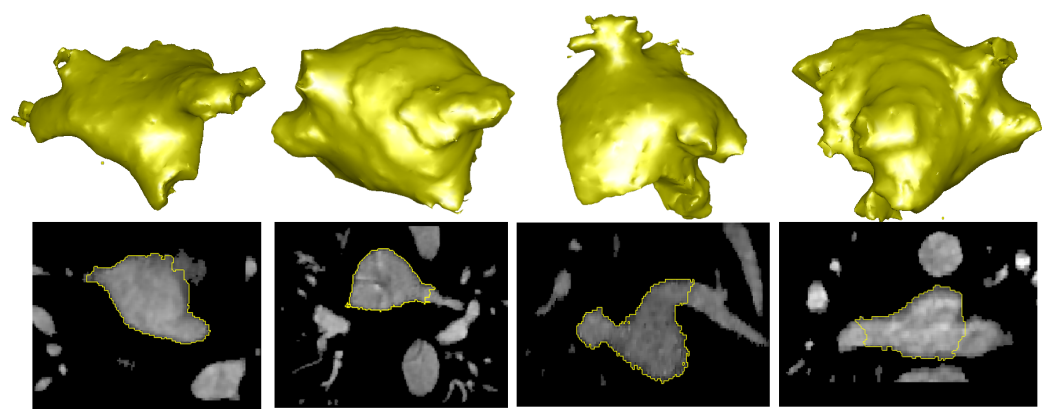

Fig. 6. (Top row) 3D surface rendering of segmented atria obtained from the proposed approach. (Bottom row) The outlines of the segmentations on the angiography images

for segmentation $[4,7,1]$ mostly use ad-hoc methods for separating the atrium from surrounding structures. The exclusion of the ventricle, the extensive vessel drainage network and the pulmonary arteries makes our approach unique. Without prior information of the anatomy, it is difficult to compute a segmentation that only obtains the clinically relevant structures which are the atrial chamber and the drainages as far as the first bifurcation. Our segmentations have shown that only structures of interest can be obtained for different anatomical configurations. In all the cases the ventricle, pulmonary arteries and the drainage networks were excluded. The incorporation of a probabilistic spatial atlas provides the model with an effective prior. Comparison with manual segmentations indicate positive results.

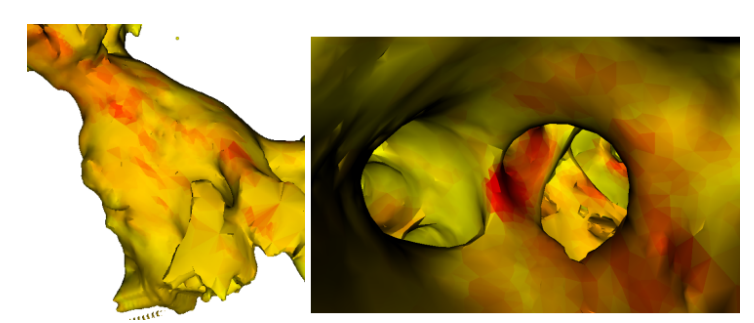

Fig. 7. Left atrial scar information can be mapped and fused to the extracted atrial geometry (left). Endocardial view (right)

The atrial geometry obtained from the segmentations are important for Electrophysiology (EP) mapping systems such as Ensite NavX and Biosense CARTO. The MRI segmentations can be loaded during a procedure to provide a more detailed anatomy than the one constructed on-site using a catheter. Also, new research into atrial fibrosis and scarring has made it essential to map scar (from pre-procedural imaging) and intra-procedural voltage information to the anatomy (see Fig. 7). Studying the temporal process of scar formation is pro- 
viding new insights into the progression and recovery of the disease process [11]. With a better atlas, the method is promising and has the potential of being applied to other imaging modalities such as free-breathing non-contrast MRA, a new recent technique in imaging.

\section{References}

1. John, M. and Rahn, N.: Automatic Left Atrium Segmentation by Cutting the Blood Pool at Narrowings. In: Proc. of MICCAI (2005)

2. Lorensen, W.E. and Cline, H.E.: Marching cubes: A High Resolution 3D Surface Construction Algorithm. In: Proc. of the 14th Annual Conference on Computer Graphics and Interactive Techniques (1987).

3. Boykov, Y., Veksler, O. and Zabih, R.: Fast Approximate Energy Minimization via Graph Cuts. In IEEE Tran. on Patt. Analysis and Machine Intelligence. 23(11). pp. 1222-1239 (2001)

4. Karim, R. and et al.: Left Atrium Segmentation for Atrial Fibrillation Ablation. In: Proceedings of SPIE Medical imaging, pp. 95. Vol. 7261. Florida (2009)

5. Marom, E.M. and et al.: Variations in Pulmonary Venous Drainage to the Left Atrium. Radiology. 230(3), pp. 824-829 (2004)

6. Greig, DM and Porteous, BT and Seheult, AH.: Exact Maximum a posteriori Estimation for Binary Images. In Jour. of the Royal Stat. Society. 51(2). 271-279 (1989)

7. Cristoforetti, A. and Faes, L. and Ravelli, F. and Centonze, M. and Del Greco, M. and Antolini, R. and Nollo, G. Isolation of the left atrial surface from cardiac multi-detector CT images based on marker controlled watershed segmentation. In Medical Engineering and Physics. 30(1). pp 48-58. (2008).

8. Meyer, C. and Manzke, R. and Peters, J. and Ecabert, O. and Kneser, R. and Reddy, V.Y. and Chan, R.C. and Weese, J. Automatic Intra-operative Generation of Geometric Left Atrium/Pulmonary Vein Models from Rotational X-Ray Angiography. In Proceedings of MICCAI 2008.

9. Hartkens, T. and Rueckert, D. and Schnabel, J.A. and Hawkes, D.J. and Hill, D.L.G. VTK CISG Registration Toolkit: An Open Source Software Package for Affine and Non-rigid Registration of Single and Multimodal 3D Images. In Bildverarbeitung für die Medizin. pp 409-412. (2002)

10. Benjamin, E.J. and Wolf, P.A. and D'Agostino, R.B. and Silbershatz, H. and Kannel, W.B. and Levy, D. Impact of Atrial Fibrillation on the Risk of Death: The Framingham Heart Study. Circulation, 98(10) (1998)

11. Oakes, R.S. and Badger, T.J. and Kholmovski, E.G. and Akoum, N. and Burgon, and others. Detection and Quantification of Left Atrial Structural Remodeling with Delayed-enhancement Magnetic Resonance Imaging in patients with Atrial Fibrillation. Circulation, 6(2) (2009)

12. Raya, S.P. and Udupa, J.K. Shape-based Interpolation of Multidimensional Objects. IEEE Trans. Med. Imaging. 9(1), pp. 32-42. (1990)

13. Otsu, N. A threshold selection method from gray-level histogram. IEEE Transactions on Systems, Man and Cybernetics 9(1). pp. 62-66. (1979) 